\title{
XXVIII.
}

\section{Ueber die chemische Zusammensetzung des Schweineharns.}

\author{
Von Dr. Georg Salomon, \\ Privatdocenten in Berin.
}

Der Harn der Schweine ist bisher nur selten chemisch untersucht worden. Die vorhandene Literatur beschränkt sich auf drei ältere, den normalen Harn betreffende Arbeiten von Lassaigne ${ }^{1}$ ), Boussingault ${ }^{2}$ ) und v. Bibra ${ }^{3}$ ) und eine aus dem Jahre 1876 stammende Mittheilung von Domenico Pecile ${ }^{4}$ ), welche letztere sich mit der Zusammensetzung des Harns bei einem gichtkranken Schwein beschäftigt. Die Angaben dieser Autoren lassen in mancher Beziehung eine Verwandtschaft zwischen dem Stoffwechsel des Schweines und dem des Menschen erkennen, wie dies auch bei der gemischten Ernährungsweise der Schweine von vornherein zu erwarten war. Ein wesentlicher Bestandtheil des menschlichen Harns wurde indessen von allen drei Untersuchern vermisst, nehmlich die Harnsäure. Das Fehlen der Harnsäure erscheint höchst auffallend, wenn man sich der fast allgemeinen Verbreitung dieses Körpers sowohl bei höheren wie bei niederen Thieren erinnert. Dennoch hat man gegen die Thatsache bisher nirgends Zweifel erhoben, sondern sich mit der Annahme zu helfen gesucht, dass die Harnsäure beim Schwein durch einen ihr nahe verwandten Stoff, das Guanin, ersetzt werde. Das Guanin war freilich nur einmal (von Pecile, l.c.) im Schweineharn aufgefunden worden. Schon 10 Jahre früher aber hatte Virchow in den Muskeln und Gelenken eines Schweineschinkens Ablagerungen von Guanin nachgewiesen und

1) Journal de pharmacie et des sciences accessoires. T. V. p. 174-176. Paris 1819.

2) Ann. de chim. et de phys. 3me sér. T.XY. p. 97-114.

3) Annalen der Chem. u. Pharm. Bd.53. S. 98-112.

4). Annalen der Chem. u. Pharm. Bd. 183. S. $14 \mathrm{i}$. 
für diesen Zustand den Namen der Guaningicht in Vorschlag gebracht ${ }^{1}$ ), so dass es allerdings nahe lag, dem Guanin eine ähnliche Rolle zuzuschreiben, wie sie die Harnsäure beim Menschen spielt.

Es ist nun aus den Arbeiten von E. Salkowski²), R. Maly und K. B. Hofmann ${ }^{3}$ ) bekannt, dass die Ausfällung der Harnsäne mittelst Salzsüure, die früher allgemein gebräuchliche Methole, nicht selten ihre Dienste vollständig versagt $\left.{ }^{2}\right)$. Ich hatte bei früheren Untersuchungen mehrfach Gelegenheit gehabt, mich von der Richtigkeit dicser Angabe zu überzeugen. So konnte ich bei einem gichtkranken Manne, demselben, an dem ich meine Untersuchungen über das Vorkommen der Harnsäure im Blute anstellte ${ }^{4}$ ), trotz annähernd normaler täglicher Harnsäureausscheidung niemals eine Fällung mit Salzsäure erhalten. In jenem und in manchen anderen Fällen hatte sich mir die von E. Salkowski angegebene Methode der Silberfällung ${ }^{5}$ ) hülfreich erwiesen. Ich beschloss deshalb, in der Voraussetzung, dass den Angaben der älteren Autoren ein Fehler der Methode zu Grunde liegen müsste, dieses Verfahren auch auf den Schweineharn anzuwenden. Gleichzeitig glaubte ich die Gelegenheit za einer Untersuchung auf Xanthinkörper, welche sich mit der Darstellung der Harnsäure leicht verbinden lässt, nicht ungenutzt vorbeigehen lassen zu dürfen. Es handelte sich dabei vor Allem um die Beantwortung der Frage, ob das Guanin auch im normalen Schweineharn vorkommt, und eventuell um die Anstellung einiger von Pecile verabsäumten Reactionen.

Es standen mir $5 \frac{1}{2} 1$ Urin zur Verfügung, die beim Schlachten aus den Blasen von 40 Schweinen durch Ausstreichen mit der Hand gewonnen worden waren. Die Thiere hatten ihr letztes Futter, das in einem Trank von Roggenkleie bestand, 16 bis 22 Stunden vor dem Tode erhalten. Die thierärztliche Unter-

1) Aren. f. pathol. Anat. Bd. 35. S. 358 u. Bd. 36. S. 147.

2) Pflüger's Archiv Bd.V. S. 210.

3) Ebendas. Bd. VI. S. 201.

4) Chajité-Annalen. V.Jahrg. (1878.) S. 137-142. Auch Salkowski beobachtete die fragliche Anomalie häufig boi Irthritikem (Lehre vow Larn S. 93).

э) Salkowski u. Lenbe, Die Lehre vom IIan. S. 96 . 
suchung vor und nach dem Tode hatte bei keinem eine Abnormität ergeben. Der Urin war blutfrei, klar, von dunkelgelber Farbe und deutlich saurer Reaction; das specifische Gewicht betrug 1021.

Nachdem durch Zusatz von Ammoniak eine beträchtliche Menge von Erdphosphaten entfernt worden war, wurde mit salpetersaurem Silber gefällt und filtrirt. Das Filtrat wurde zu weiterer Untersuchung aufbewahrt. Der Silberniederschlag wurde mit Schwefelwasserstoff zerlegt, das zur Trockne gedampfte Filtrat mit warmer verdünnter Schwefelsüure (1:30) aufgenommen und nach längerem Stehen filtrirt. Es hinterblieb ein ganz beträchtlicher Rückstand, der jedoch nicht ganz frei von anorganischen Verunreinigungen war und deswegen nochmals mit verdünnter warmer Salzsäure extrahirt wurde. Die salzsaure, sowie die schwefelsaure Lösung wurden zur weiteren Prüfung zurückgestellt. - Der auf diese Weise gereinigte, gut gewaschene Rückstand, der nichts Anderes sein konnte als Harnsäure, wurde in Natronlauge gelöst and durch Erwärmen mit verdünnter Salzsüure wieder ausgefällt. Ich erhielt so $0,65 \mathrm{~g}$ eines gut krystallisirten, aschefreien, fast weissen Präparates, das sich durch Murexidprobe und Elementaranalyse als Harnsäure auswies. 0,2480 Substanz gaben $0,3290 \mathrm{CO}_{2}=36,17$ pCt. $\mathrm{C}$ and $0,0674 \mathrm{H}_{2} \mathrm{O}$ $=2,72$ pCt. $\mathrm{H}$.

0,1020 Substanz gaben nach Dumas $29,5 \mathrm{ccm}$ Stickstoff bei $18,8^{\circ}$ und $754,0 \mathrm{~mm}$ Druck ( $=33,07$ pCt. $\mathrm{N}$ ).

$\begin{array}{lrr} & \text { Berechnet. } & \text { Gefunden. } \\ \mathrm{C}_{5} & 35,71 & 36,17 \text { pCt. } \\ \mathrm{H}_{4} & 2,38 & 2,72- \\ \mathrm{N}_{4} & 33,33 & 33,07- \\ \mathrm{O}_{3} & 28,58 & -\end{array}$

Die Menge der Harnsäure beim Schwein würde hiernach allerdings hinter der des menschlichen Harns beträchtlich zurückbleiben, immerhin aber die in anderen Thierklassen, z. B. bej den Raubthieren und bei Pflanzenfressern vorkommende noch übertreffen. Ihr Verhältniss zum Harnstoff liess sich bei dem einmal gewählten Gange der Untersuchung nicht feststellen, sondern musste an einer neuen Harnportion ermittelt werden. Es wurden daher noch 21 Schweineharn auf dieselbe Weise und unter genau denselben Ernährungsverhältnissen wie das erste Archiv f. pathol. Anat. Bd. XCV. Het. 3 . 
Yal aufgesammelt. Der Trin war auch diesmal klar, dunkelgelb und von stark saurer Reaction; das specifische Gewicht betrug 1024. Er eathielt 4,1 pCt. Harnstoff und gab, entsprechend dieser hohen Concentration, schon bei directen Zusatz von Salpetersäure reichliche Vengen von salpetersaurem Harnstoff. Die Menge der Harnsäure, diesmal genau nach dem von Salkowski angegebenen Verfahren bestimmat, betrug $0,0265 \mathrm{pCt}$, während mit Salzsäure wiederum kein Niederschlag erzielt wurde. Hieraus würde sich ein Verhältniss der Harnsäure zam Harnstoff von 1:150 ergeben; ob dasselhe als Durchschnittsmaass betrachtet werden darf, lässt sich natürlich nicht entscheiden.

Die schwefelsaure Lösung (vgl. oben) wurde mit Ammoniak übersättigt, mit Silber gefällt und der Niederschlag nach Neubauer in heisser Salpetersäure von 1,1 spec. Gew. gelöst. Beim Erkalten schied sich ein Niederschlag ans, der abfiltrirt und nach mehrmaligem Umkrystallisiren aus heisser Salpetersäure mit Schwefolwasserstoff zerlegt wurde. Das mit Ammoniak versetzte Filtrat vom Schwefelsilber trübte sich beim Einengen bald durch Ausscheidung schöner, fast millimeterlanger Nadeln und Platten. Das salpetersaure Filtrat lieferte nach Uebersättigen mit Ammoniak, Zerlegen des entstandenen Niederschlages mit Schwefelwasserstoff und Eindampfen einen in sehr kleinen mikroskopischen, garbenförmig angeordneten Nadeln krystallisirenden Niederschlag. Die Eigenschaften dieser beiden offenbar der Xanthingruppe angehörigen Substanzen sollen in übersichtlicher Form durch die nachfolgende Tabelle veranschaulicht werden:

\begin{tabular}{|c|c|c|c|c|c|c|}
\hline No. & $\begin{array}{l}\text { Ausschei- } \\
\text { dungsform. }\end{array}$ & An & $\begin{array}{c}\text { In } \\
\mathrm{NaHO} .\end{array}$ & $\begin{array}{l}\text { Mit Blei- } \\
\text { zucker. }\end{array}$ & $\begin{array}{l}\text { Mit Blei- } \\
\text { essig. }\end{array}$ & $\begin{array}{l}\text { Mit Bleiessig } \\
\quad+\mathrm{NH}_{3} \text {. }\end{array}$ \\
\hline I. & $\begin{array}{c}\text { Makroskop. } \\
\text { Krystalle }\end{array}$ & uniöslich & löslich & 0 & 0 & $\begin{array}{l}\text { weisser } \\
\text { Niederschlag }\end{array}$ \\
\hline L. & $\begin{array}{c}\text { Mikroskop. } \\
\text { Kiystalle }\end{array}$ & löslich & löslich & 0 & 0 & $\begin{array}{c}\text { weisser } \\
\text { Niederschlag }\end{array}$ \\
\hline
\end{tabular}

\begin{tabular}{|c|c|c|c|c|c|c|}
\hline $\begin{array}{l}\text { Mit Queck- } \\
\text { silbersalzen. }\end{array}$ & $\mid \begin{array}{c}\text { Mit Pi- } \\
\text { krinsäure. }\end{array}$ & Verbind. $n$ & $\begin{array}{l}\text { it } \mathrm{AgNO}_{3} \text {. } \\
\text { in Salpe- } \\
\text { tersäure. }\end{array}$ & $\begin{array}{l}\text { Xanthin } \\
\text { reaction. }\end{array}$ & $\begin{array}{l}\text { Weidel- } \\
\text { scheReact. }\end{array}$ & $\begin{array}{c}\text { Beim } \\
\text { Erbitzen. }\end{array}$ \\
\hline $\begin{array}{c}\text { weisser } \\
\text { Niederschlag } \\
\text { weisser } \\
\text { Niederschlag }\end{array}$ & $\begin{array}{c}\text { gelbe } \\
\text { Krystalle } \\
0\end{array}$ & $\begin{array}{l}\text { krystallis. } \\
\text { krystallis. }\end{array}$ & $\begin{array}{l}\text { unlöslich } \\
\text { löslich }\end{array}$ & $\begin{array}{l}\text { stank } \\
\text { stark }\end{array}$ & $\begin{array}{c}0 \\
\text { Rothfitr- } \\
\text { bung }\end{array}$ & $\begin{array}{c}\text { Isonitril- } \\
\text { geruch } \\
0\end{array}$ \\
\hline
\end{tabular}


Besondere Aufmerksamkeit verdient der erste von diesen Körpern, weil sein Verhalten in allen wesentlichen Punkten mit dem des Guanins übereinstimmt. Beachtenswerth und für die Unterscheidung vom Hypoxanthin verwerthbar sind vor Allem die Unlöslickkeit in Ammoniak, die Bildung intensiv gelber Krystalle mit Pikrinsäure und die stark ausgesprochene Xanthinreaction bei fehlender Weidel'scher Reaction. (Die letzter besteht in einer Rothfärbung beim Eindampfen mit Chlorwasser and Salpetersäure und nachfolgender Einwirkung von Ammoniak.) Hypoxanthin ist in Ammoniak leicht löslich; es liefert beim Zasatz von Pikrinsäure zur salzsauren Lösung keine intensiv gelben, sondern kaum gefärbte Krystalle (Capranica); es giebt die gewöhnliche Xanthinreaction mit Salpetersäure und Natronlauge nur sehr schwach (E. Salkowski). Das Ausbleiben der Weidel'schen Reaction würde sowohl für Hypoxanthin wie für Guanin sprechen; doch deutet eben die gleichzeitig vorhandene starke Xanthinreaction entschieden auf letzteren Körper hin. Die Krystallisationsfähigkeit spricht nicht unbedingt gegen Guanin, da nach Drechsel (Journ. f. pract. Chem. Bd. 24. S. 44) das bisher meist für amorph gehaltene Guanin unter Umständen auch krystallisirt erhalten werden kann. Nach dem angewandten Verfahren wäre auch die Möglichkeit nicht ausgeschlossen, dass sich während der Darstellung die von Kossel ${ }^{1}$ ) beschriebene krystallisirende Verbindung des Guanins mit Ammoniak gebildet haben könnte. Nach einer von Krukenberg und Wagner ${ }^{2}$ ) aufgestellten Reactionstabelle würde allerdings dem Guanin noch eine Eigenschaft zukommen, die bei meinem Präparat nicht vorhanden war, nehmlich die Fällbarkeit durch neutrales und basisch essigsaures Bleioxyd. Ich habe mich indessen von der Richtigkeit dieser Angabe nicht überzeugen können. Soviel mir bekannt, sind sowohl Hypoxanthin wie Guanin durch die Bleisalze nur bei gleichzeitigem Zusatz von Ammoniak fällbar.

Allen den Indicien, die mit so grosser Entschiedenheit für das Vorhandensein von Guanin im normalen Schweineharn zu sprechen scheinen, steht aber vor der Hand noch eine Reaction gegenüber, die meiner Erfahrung nach dem Guanin nicht zu-

1) Zeitschr. f. physiol. Chem. Bd. VII. Hft. 1.

2) Sitzb. der Würzburger phys.-med. Ges. 1883. 
kommt; die Entwicklung eines Isonitrilyeruches (oder vielleicht besser: "isonitrilähnlichen Geruches") beim Erhitzen auf dem Platinblech. Da Pecile über das Verhalten des von ihm gefundenen Körpers beim Erwärmen keine Angaben macht, so lässt sich nicht wohl entscheiden, ob er mit dem meinigen identisch war oder sich wie gewöhnliches Guanin verhielt. Bemerken will ich nur, dass Pecile's Nachweis des Guanins, der sich hauptsächlich auf eine Analyse der salpetersauren Silberverbindung stützt, insofern eine wesentliche Lücke erkennen lässt, als er über die Lösliohkeit oder Tnlöslichkeit seiner Substanz in Ammoniak keine Auskunft giebt. Da ich noch weniger Material in Händen hatte als Pecile, nehmlich nur $0,02 \mathrm{~g}$ reiner Substanz, so war mir leider die Möglichkeit, Analysen der Salze, geschweige denn Elementaranalysen anzustellen, von vornherein benommen.

Der zweite in der Tabelle aufgeführte Körper ist jedenfalls als Xanthin zu bezeichnen. Ich möchte hierfür besonders die Rothfärbung mit Chlorwasser und Salpetersäure geltend machen, eine Reaction, die ich trotz der von Krukenberg und Wagner (l. c.) erhobenen Einwände in Vebereinstimmung mit Kossel dem Xanthin zuschreiben muss. Auch Pecile hat neben dem Guanin Xanthin gefunden.

Die salzsaure Lösung (s. oben) enthielt wider Erwarten, wiewohl eine gründliche Extraction mit verdïnnter Schwefelsüure vorangegangen war, neben Mineralbestandtheilen noch zwei Substanzen, die sich durch die Xanthinreaction, Fällbarkeit durch ammoniakalische Silberlösung, Bildung von Doppelsalzen mit salpetersaurem Silber a. s. w. als Xanthinkörper .charakterisirten. Ich habe mich mit denselben nicht weiter beschäftigt.

Ausser der Harnsäure und den Xanthinkörpern fand ich in dem Harn noch Kreatin, das sich vielleicht aus ursprünglich vorhandenem Kreatinin gebildet hatte, und eine in Aether lösliche Säure. Ersteres wurde aus dem alkoholischen Auszug der vom ersten Silberniederschlage abfiltrirten, zum Syrup gedampften Flüssigkeit (s. oben S. 529 Z. 6) gewonnen. Nachdem der Alkohol verdunstet und der zurückbleibende, zumeist aus Harnstoff bestehende Krystallbrei mit Wasser aufgenommen worden war, blieb das Kreatin in Form gelblicher sandartiger Krystallkörner 
ungelöst zurïck. Der Nachweis warde nach Ueberführung in Kreatinin darch die Weyl'sche Reaction mit Nitroprussidnatrium und Natronlauge und durch die Herstellung von Chlorzinkkreatinin geführt. Zur ersten Erkennung diente mir eine Geruchsreaction, die ich gelegentlich beim reinen Kreatin bemerkt und bereits öfter mit Nutzen angewendet habe. Die Substanz entwickelte nehmlich beim Erhitzen auf dem Platinblech weisse 1)ämpfe von eigenthümlich durchdringendem, einigermaassen an Knoblauch erinnerndem Geruch. - Die oben erwähnte Säure wurde nach dem zur Darstellung der Milchsäure üblichen Verfahren zunächst in Form eines krystallisirenden Zinksalzes erhalten; die Ausbeute betrug $0,04 \mathrm{~g}$. Es lag in der That nahe, das Product für milchsaures Zink zu halten, da von Boussingault (l. c.) die Milchsäure unter den Bestandtheilen des Schweineharns aufgeführt wird. Indessen war von vornherein durch die Schwerlöslichkeit des Zinksalzes Fleischmilchsäure ausgeschlossen. Da es sich ferner herausstelite, dass kein Krystallwasser vorhanden war, so war ein weiteres wichtiges Argument gegen Milchsäure überhaupt gegeben (fleischmilchsaures Zink enthält circa 12, gährungsmilchsaures circa 18 pCt. Krystallwasser). Endlich wurde, nachdem die Verbindung mit Schwefelwasserstoff zerlegt war, durch die vollkommene Krystallisationsfähigkeit der Säure jeder Gedanke an Milchsäure ausgeschlossen. Einen positiven Aufschluss über ihre Natur habe ich nicht erhalten können; ich will nur erwähnen, dass sie beim Erhitzen heftig zum Husten reizende Dämpfe entwickelte, eine Eigenschaft, die vielleicht auf Bernsteinsäure hindeutet. - Für die Gegenwart von Hippursäure im Schweineharn habe ich zwar bisher keinerlei Anzeichen gefunden, möchte dieselbe aber noch nicht unbedingt ausschliessen.

Obige Untersuchungen sind in dem unter Leitung des Herrn Professor E. Salkowski stehenden Laboratorium des Berliner pathologischen Instituts ausgeführt worden.

Nach Abschluss dieser Arbeit finde ich in einer Arbeit von Meissl und Strohmer: "Ueber die Bildung von Fett aus Kohlehydraten irn Thierkörper" (Ber. d. Wiener Akad. 1883, Abth. III. S. 205) eine Notiz über das Vorkommen von Hippur- 
säure und Harnsäure im Schweineharn. Der betreffende Passus lautet: ,Thatsächlich konnte aus dem mit Kalilauge eingedampften Harn nach dem Ansäuern durch Extraction mit Aether Benzoësäure isolirt werden; der Harn enthielt demnach Hippursäure: die directe Prüfung darauf ergab blos ein zweifelhaftes Resultat. Ferner liessen sich Spuren von Harnsäure qualitativ nachweisen" (1. c. S. 214). Weitere Angaben fehlen.

\section{XXIX.}

\section{Beiträge zur Kenntniss der Trichinosis und der Actinomycosis bei Schweinen.}

Von Rud. Virchow.

1. Amerikanische Trichinen.

Das Vorkommen von Trichinen beim Schweine ist in Nordamerika zuerst beobachtet worden. Wie ich in meiner kleinen Schrift (Die Lehre von den Trichinen. 3. Aufl. 1866. S.5 u. 36) erwähnt habe, fand sie Jos. Leidy schon 1847. Dass auch schon längst Uebertragungen auf den Menschen vorkamen, erhellt aus den Funden desselben Forschers, sowie denen von Bowditch, der einmal in einem Stück Menschenfleisch von $\frac{1}{4} \square$ Zoll Oberfläche 50 Stück beobachtete und daraus für das ganze Individuum über $7 \frac{1}{2}$ Millionen Stück berechnete (Samuel D. Gross, Elements of pathol. anat. Philad. 1845. p.216). Indess diese Beobachtungen beim Menschen betrafen geheilte Fälle. So ist es denn gekommen, dass erst lange, nachdem in Europa die frische Trichinose erkannt war, in Amerika die Aufmerksamkeit sich darauf richtete. Ja, selbst die grosse Häufigkeit des Vorkommens von Trichinen bei amerikanischen Schweinen ist zuerst in Europa festgestellt worden, als man bei uns in Deutschland anfing, den Import zu controliren.

Wie weit in Amerika Schweinetrichinen verbreitet sind, ist noch jetat nicht au äbersehen. Meines Wissens ist aus Süd- 